\title{
OPTIMAL FILTERING IN DISCRETE-TIME SYSTEMS WITH TIME DELAYS AND MARKOVIAN JUMP PARAMETERS
}

\author{
CHUNYAN HAN $^{1}$ and HUANSHUI ZHANG ${ }^{\otimes 1}$
}

(Received 6 February, 2009; revised 14 December, 2009)

\begin{abstract}
This paper investigates the linear minimum mean-square error estimation for discretetime Markovian jump linear systems with delayed measurements. The key technique applied for treating the measurement delay is reorganization innovation analysis, by which the state estimation with delayed measurements is transformed into a standard linear mean-square filter of an associated delay-free system. The optimal filter is derived based on the innovation analysis method together with geometric arguments in an appropriate Hilbert space. The solution is given in terms of two Riccati difference equations. Finally, a simulation example is presented to illustrate the efficiency of the proposed method.
\end{abstract}

2000 Mathematics subject classification: primary 93E11.

Keywords and phrases: Markovian jump linear systems, time-delay systems, optimal filtering, reorganized innovation analysis, Riccati equations.

\section{Introduction}

Markov jump linear systems (MJLSs), which are defined as linear systems whose parameters evolve according to a finite-state Markov chain, are used in many applications of signal processing, digital communications and target tracking (see [14] and the references therein). In the past decade, the optimal control and stabilization problems have been extensively studied for MJLSs [5, 10]. Also, the filtering problem of systems with jumping parameters has recently begun to receive attention. There are several methods of achieving the state estimation of MJLSs which differ in their estimation criteria and means. The linear minimum mean-square error (LMMSE) filter $[9,11]$ and the interacting multiple-model methods [6] are the most famous of the filtering algorithms. The LMMSE formulation leads to a time-varying linear filter that

\footnotetext{
${ }^{1}$ School of Control Science and Engineering, Shandong University, Jingshi Road 73, Jinan 250061, PR China; e-mail: cyhan823@hotmail.com, hszhang@sdu.edu.cn.

(C) Australian Mathematical Society 2010, Serial-fee code 1446-1811/2010\$16.00
} 
is easy to implement, in which all calculations can be performed off-line, and therefore it is an efficient and popular estimation tool. Other existing algorithms include the classical generalized pseudo-Bayesian filter [1], MAP estimation based approaches and the stochastic sampling based methods [7]. It should be noted that almost all existing methods focus on the study of state estimation for MJLSs without delay.

Time delay occurs frequently in many practical systems, such as manufacturing, telecommunications and economic systems. When the systems have delays in their dynamics, the filtering and control problems become difficult to solve. For the class of deterministic linear time-delay systems, there exist many papers in the literature; see $[3,4,17,18]$ and the references therein. For the case of MJLSs with delay terms, robust filtering and robust $H_{\infty}$ filtering methods have been designed in [8, 15, 16] by using the linear matrix inequality tool. However, in these papers, only state delay systems with Markovian switching were studied. To the best of the authors' knowledge, the problem of state estimation for MJLSs with observation delays has not received much attention in the past. The purpose of this paper is to extend some results on the state estimation of the class of linear time-delay systems [18] to the MJLS with delayed observations.

In this paper, we study the LMMSE estimation for discrete-time MJLSs with observation delays by using the reorganized innovation analysis method. With this method, the state estimation with delayed measurements for MJLSs can be converted into that of a standard delay-free system. A finite-dimensional recursive filter is then obtained in terms of Riccati difference equations based on the geometric arguments in the Hilbert space. It will be shown that, different from the standard delay-free system, the optimal filtering formulation for time-delay MJLS model consists of two finite linear filters with dimension $N^{2} n$, where $n$ denotes the dimension of the state vector, and $N$ is the number of the states of the Markov chain.

The rest of this paper is organized as follows. In Section 2 we present the problem formulation and some assumptions. In Section 3 the optimal filter is derived by using reorganized innovation analysis and geometric arguments. In Section 4 a simulation example is constructed to illustrate the main results. Finally, some concluding remarks are made in Section 5.

The following notation is fairly standard. $\mathbb{R}^{n}$ denotes the $n$-dimensional Euclidean space. $\mathbb{B}\left(\mathbb{R}^{n}, \mathbb{R}^{m}\right)$ denotes the norm bounded linear space of all $m \times n$ matrices, with $\mathbb{B}\left(\mathbb{R}^{n}\right)=\mathbb{B}\left(\mathbb{R}^{n}, \mathbb{R}^{n}\right)$. For $L \in \mathbb{B}\left(\mathbb{R}^{n}\right), L^{\prime}$ indicates the transpose of $L$, and $L \geq 0(L>$ 0 ) means that the symmetric matrix $L$ is positive semi-definite (positive definite). For a collection of $N$ matrices $D_{1}, \ldots, D_{N}$, with $D_{j} \in \mathbb{B}\left(\mathbb{R}^{n}, \mathbb{R}^{m}\right), \operatorname{diag}\left\{D_{j}\right\} \in$ $\mathbb{B}\left(\mathbb{R}^{N n}, \mathbb{R}^{N m}\right)$ represents the diagonal matrix formed by $D_{j}$ in the diagonal and zero elsewhere. Similarly, for a set of $N^{2}$ matrices $D_{11}, D_{1 N}, \ldots, D_{N 1}, \ldots, D_{N N}$ with $D_{i j} \in \mathbb{B}\left(\mathbb{R}^{n}, \mathbb{R}^{m}\right), \operatorname{diag}\left\{D_{i j}\right\} \in \mathbb{B}\left(\mathbb{R}^{N^{2} n}, \mathbb{R}^{N^{2} m}\right)$ denotes the diagonal matrix formed by $D_{i j}$ in the diagonal and zeros elsewhere. In addition, $\mathbf{1}_{\{\cdot\}}$ stands for the Dirac measure, $\mathrm{E}(\cdot)$ represents the expected value operator, $\operatorname{col}(\cdot)$ denotes the column vector, and $\operatorname{cov}(\cdot)$ means covariance. 


\section{Problem formulation}

On a probability space $(\Omega, \mathcal{P}, \mathcal{F})$ we consider the Markov jump linear system with delayed measurements

$$
\begin{aligned}
x(k+1) & =A_{\theta(k)} x(k)+C_{\theta(k)} w(k), \\
y(k) & =H_{\theta(k)} x(k)+G_{\theta(k)} v(k), \\
y_{1}(k) & =\tilde{H}_{\theta(k)} x(k-d)+\tilde{G}_{\theta(k)} v_{1}(k),
\end{aligned}
$$

where $x(k) \in \mathbb{R}^{n}$ denotes the state vector, $y(k) \in \mathbb{R}^{m}$ and $y_{1}(k) \in \mathbb{R}^{m_{1}}$ are respectively the instantaneous and delayed measurements, $w(k) \in \mathbb{R}^{r}, v(k) \in \mathbb{R}^{p}$ and $v_{1}(k) \in \mathbb{R}^{p_{1}}$ are random disturbances. Further, $d$ is the measurement delay which is an integer and $\theta(k)$ is a discrete-time Markov chain taking values in a finite state space $\{1, \ldots, N\}$, and with transition probability matrix $\Lambda=\left[\left(\lambda_{i j}\right)\right]$. We set $\pi_{i}(k)=\mathcal{P}(\theta(k)=i)(i=1, \ldots, N)$ and denote $\pi(k)=\left[\pi_{1}(k) \cdots \pi_{N}(k)\right]^{\prime}$, where $\pi(k)$ satisfies the Kolmogorov forward difference equation $\pi(k+1)=\Lambda^{\prime} \pi(k)$. The matrices $A_{i}, C_{i}, H_{i}, G_{i}, \tilde{H}_{i}$ and $\tilde{G}_{i}(i=1, \ldots, N)$ are of appropriate dimensions. We shall make the following assumptions.

ASSUMPTION 1. $\{w(k)\},\{v(k)\}$ and $\left\{v_{1}(k)\right\}$ are zero-mean second-order, independent wide-sense stationary sequences with covariance matrices equal to the identity. $\{w(k)\},\{v(k)\}$ and $\left\{v_{1}(k)\right\}$ are mutually independent.

ASSUMPTION 2. The initial state $x(0)$ is also a second-order random vector with zero mean and covariance matrix $V$.

ASSUMPTION 3. $x(0)$ and $\{\theta(k)\}$ are independent of $\{w(k)\},\{v(k)\}$ and $\left\{v_{1}(k)\right\}$.

AsSumption 4. For all $i, j=1,2, \ldots, N, G_{i} G_{i}^{\prime}>0$ and $\tilde{G}_{j} \tilde{G}_{j}^{\prime}>0$.

In view of Assumptions 1 and 2, it is obvious that $x(k), y(k)$ and $y_{1}(k)$ are sequences of second-order random vectors, and $\mathrm{E}(y(k))=0, \mathrm{E}\left(y_{1}(k)\right)=0$. At time $k$ let $y_{d}(k)$ denote the observation of system (2.2)-(2.3) and $v_{d}(k)$ denote the related observation noise. Then

$$
y_{d}(k)= \begin{cases}y(k), & 0 \leq k \leq d, \\ \operatorname{col}\left\{y(k), y_{1}(k)\right\}, & k \geq d,\end{cases}
$$

and

$$
v_{d}(k)= \begin{cases}v(k), & 0 \leq k \leq d, \\ \operatorname{col}\left\{v(k), v_{1}(k)\right\}, & k \geq d .\end{cases}
$$

We set $\mathcal{F}_{k}$ to be the $\sigma$-field generated by $\left\{x(s), y_{d}(s), \theta(s) ; s=0, \ldots, k\right\}$, and denote by $\mathcal{L}\left\{\left.y_{d}(s)\right|_{0 \leq s \leq k}\right\}$ the linear space spanned by the observations $y_{d}(s), s=0, \ldots, k$. For any $r, \eta \in \mathcal{L}\left\{\left.y_{d}(s)\right|_{0 \leq s \leq k}\right\}$, the inner product $\langle\cdot ; \cdot\rangle$ in $\mathcal{L}\left\{\left.y_{d}(s)\right|_{0 \leq s \leq k}\right\}$ is given by $\langle r ; \eta\rangle=\mathrm{E}\left(r \eta^{\prime}\right)$, and therefore $r$ and $\eta$ are orthogonal if $\langle r ; \eta\rangle=0$. In addition, 
for any second-order random vector $r(k)$, we shall denote by $\hat{r}(k \mid s)$ the estimator of $r(k)$ given $\mathcal{L}\left\{\left.y_{d}(s)\right|_{0 \leq s \leq k}\right\}$.

The estimation problem of this paper can be stated as follows: given the observations $\left\{\left.y_{d}(s)\right|_{0 \leq s \leq k}\right\}$, find an LMMSE estimator $\hat{x}(k \mid k)$ of $x(k)$.

In view of the geometric arguments as in [12], the LMMSE estimator $\hat{x}(k \mid s)$ has the following properties:

(1) $\hat{x}(k \mid s) \in \mathcal{L}\left\{\left.y_{d}(s)\right|_{0 \leq s \leq k}\right\}$

(2) $x(k)-\hat{x}(k \mid s)$ is orthogonal to $\mathcal{L}\left\{\left.y_{d}(s)\right|_{0 \leq s \leq k}\right\}$.

Furthermore, the filtering equations can be defined as

$$
\begin{aligned}
\hat{x}(k \mid s)= & \mathrm{E}\left(x(k)\left(\left(y_{d}\right)^{s-1}\right)^{\prime}\right) \operatorname{cov}\left(\left(y_{d}\right)^{s-1}\right)^{-1}\left(y_{d}\right)^{s-1}, \\
\hat{x}(k \mid k)= & \mathrm{E}\left(x(k) \tilde{y}_{d}(k \mid k-1)^{\prime}\right) \mathrm{E}\left(\tilde{y}_{d}(k \mid k-1) \tilde{y}_{d}(k \mid k-1)^{\prime}\right)^{-1}\left(y_{d}(k)-\hat{y}_{d}(k \mid k-1)\right) \\
& +\hat{x}(k \mid k-1),
\end{aligned}
$$

where

$$
\left(y_{d}\right)^{s-1}=\operatorname{col}\left\{y_{d}(0), \ldots, y_{d}(s-1)\right\} \quad \text { and } \quad \tilde{y}_{d}(k \mid k-1)=y_{d}(k)-\hat{y}_{d}(k \mid k-1) .
$$

\section{Optimal estimation}

In this section we shall present the solution to the above optimal estimation problem by reorganizing the innovation sequences and applying the projection in the Hilbert space. For simplicity of discussion, we suppose that the time $k>d$, and denote $k_{1}=k-d$. The case $0 \leq k \leq d$ can be discussed in the same manner.

3.1. Reorganized observations In order to solve such problem caused by delay, we shall define another observation sequence which is delay-free and spans the same linear space as the one generated by the original observation. To this end, let

$$
\begin{array}{ll}
\bar{y}_{2}(s) \triangleq \operatorname{col}\left\{y(s), y_{1}(s+d)\right\}, & 0 \leq s \leq k_{1}, \\
\bar{y}_{1}(s) \triangleq y(s), & k_{1}<s \leq k .
\end{array}
$$

It is clear that

$$
\begin{array}{ll}
\bar{y}_{2}(s)=\bar{H}_{\theta(s)} x(s)+\bar{G}_{\theta(s)} \bar{v}(s), & 0 \leq s \leq k_{1}, \\
\bar{y}_{1}(s)=H_{\theta(s)} x(s)+G_{\theta(s)} v(s), & k_{1}<s \leq k,
\end{array}
$$

where

$$
\bar{H}_{\theta(s)}=\left[\begin{array}{c}
H_{\theta(s)} \\
\tilde{H}_{\theta(s+d)}
\end{array}\right], \quad \bar{G}_{\theta(s)}=\left[\begin{array}{cc}
G_{\theta(s)} & 0 \\
0 & \tilde{G}_{\theta(s+d)}
\end{array}\right], \quad \bar{v}(s)=\left[\begin{array}{c}
v(s) \\
v_{1}(s+d)
\end{array}\right] .
$$

It can be seen from (3.1) that $\bar{y}_{i}(s)(i=1,2)$ is composed of different observations associated with the same state $x(s)$. Obviously, there no longer exist delays in the reorganized observation equations. Moreover, it is apparent that the following lemma is true. 
LEMMA 3.1. For the given time instant $k$, the linear space generated by $\left\{\left.y_{d}(s)\right|_{0 \leq s \leq k}\right\}$ is equivalent to the linear space of

$$
\mathcal{L}\left\{\left.\bar{y}_{2}(s)\right|_{0 \leq s \leq k_{1}} ;\left.\bar{y}_{1}(s)\right|_{k_{1}<s \leq k}\right\} .
$$

Note that the jump parameters considered in this paper are not known in advance, so we shall estimate $x(s), \theta(s)$ and $\theta(s+d)$ synchronously by introducing the new stochastic variables

$$
\begin{array}{ll}
z_{i j}(s, 2)=x(s) \mathbf{1}_{\{\theta(s)=i\}} \mathbf{1}_{\{\theta(s+d)=j\}}, & 0 \leq s \leq k_{1}, \\
z_{i}(s, 1)=x(s) \mathbf{1}_{\{\theta(s)=i\}}, & k_{1}<s \leq k .
\end{array}
$$

Let

$$
\begin{aligned}
z(s, 2)= & \operatorname{col}\left\{z_{11}(s, 2), \ldots, z_{1 N}(s, 2), z_{21}(s, 2), \ldots, z_{2 N}(s, 2), \ldots,\right. \\
& \left.z_{N 1}(s, 2), \ldots, z_{N N}(s, 2)\right\} \\
z(s, 1)= & \operatorname{col}\left\{z_{1}(s, 1), z_{2}(s, 1), \ldots, z_{N}(s, 1)\right\}
\end{aligned}
$$

Then from the definition of $z_{i j}(s, 2)$ and $z_{i j}(s, 1)$, it is clear that

$$
x(s)= \begin{cases}\sum_{i=1}^{N} \sum_{j=1}^{N} z_{i j}(s, 2), & 0 \leq s \leq k_{1}, \\ \sum_{i=1}^{N} z_{i}(s, 1), & k_{1}<s \leq k .\end{cases}
$$

In view of (3.5), it is obvious that $\hat{x}(s \mid s)$ can be obtained via the estimator of $z(s, 1)$ and $z(s, 2)$. So the idea here is, instead of directly estimating the state $x(s)$, we estimate the random vector $z(s, 2)$ and $z(s, 1)$ by taking advantage of the Markov jumping property. Write

$$
\begin{aligned}
& H=\left[H_{1} H_{2} \cdots H_{N}\right] \in \mathbb{B}\left(\mathbb{R}^{m}, \mathbb{R}^{n N}\right), \\
& \bar{H}=\left[H_{11} \cdots H_{1 N} \cdots H_{N 1} \cdots H_{N N}\right] \in \mathbb{B}\left(\mathbb{R}^{\left(m+m_{1}\right)}, \mathbb{R}^{n N^{2}}\right),
\end{aligned}
$$

with $H_{i j}=\left[\begin{array}{ll}H_{i}^{\prime} & \tilde{H}_{j}^{\prime}\end{array}\right]^{\prime}, i, j=1, \ldots, N$, and define

$$
\begin{aligned}
\bar{G}(s) & =\operatorname{diag}\{G(s), \tilde{G}(s+d)\}, & & 0 \leq s \leq k_{1}, \\
G(s) & =\left[G_{1} \pi_{1}(s)^{1 / 2} \cdots G_{N} \pi_{N}(s)^{1 / 2}\right], & & 0 \leq s \leq k, \\
\tilde{G}(s+d) & =\left[\tilde{G}_{1} \pi_{1}(s+d)^{1 / 2} \cdots \tilde{G}_{N} \pi_{N}(s+d)^{1 / 2}\right], & & 0 \leq s \leq k_{1} .
\end{aligned}
$$

Then the observation equation (3.1) can be rewritten as

$$
\begin{array}{ll}
\bar{y}_{2}(s)=\bar{H} z(s, 2)+\bar{G}_{\theta(s)} \bar{v}(s), & 0 \leq s \leq k_{1}, \\
\bar{y}_{1}(s)=H z(s, 1)+G_{\theta(s)} v(s), & k_{1}<s \leq k,
\end{array}
$$


and the covariance matrices of $\bar{G}_{\theta(s)} \bar{v}(s)$ and $G_{\theta(s)} v(s)$ can be given as

$$
\mathrm{E}\left[\bar{G}_{\theta(s)} \bar{v}(s) \bar{v}(s)^{\prime} \bar{G}_{\theta(s)}^{\prime}\right]=\bar{G}(s) \bar{G}(s)^{\prime}, \quad \mathrm{E}\left[G_{\theta(s)} v(s) v(s)^{\prime} G_{\theta(s)}^{\prime}\right]=G(s) G(s)^{\prime} .
$$

3.2. Reorganized innovation sequences To derive the Riccati difference equations of the covariance matrices of the estimation errors, we introduce the stochastic sequences

$$
\begin{array}{ll}
\varepsilon_{2}(s)=\bar{y}_{2}(s)-\hat{\bar{y}}_{2}(s \mid s-1), & 0 \leq s \leq k_{1}, \\
\varepsilon_{1}(s)=\bar{y}_{1}(s)-\hat{\bar{y}}_{1}(s \mid s-1), & k_{1}<s \leq k,
\end{array}
$$

where $\hat{\bar{y}}_{2}(s \mid s-1)$ is the projection of $\bar{y}_{2}(s)$ onto the linear space of

$$
\mathcal{L}\left\{\left.\bar{y}_{2}(\tau)\right|_{0 \leq \tau \leq s-1}\right\}
$$

and $\hat{\bar{y}}_{1}(s \mid s-1)$ is the projection of $\bar{y}_{1}(s)$ onto the linear space of

$$
\mathcal{L}\left\{\left.\bar{y}_{2}(\tau)\right|_{0 \leq \tau \leq k_{1}} ;\left.\bar{y}_{1}(\tau)\right|_{k_{1}<\tau \leq s-1}\right\} .
$$

We then can rewrite (3.7) and (3.8) as

$$
\begin{aligned}
& \varepsilon_{2}(s)=\bar{H} \tilde{z}(s, 2 \mid s-1)+\bar{G}_{\theta(s)} \bar{v}(s), \\
& \varepsilon_{1}(s)=H \tilde{z}(s, 1 \mid s-1)+G_{\theta(s)} v(s),
\end{aligned}
$$

where

$$
\tilde{z}(s, \ell \mid s-1)=z(s, \ell)-\hat{z}(s, \ell \mid s-1), \quad \ell=1,2,
$$

with $\hat{z}(s, 2 \mid s-1)$ the projection of $z(s, 2)$ onto the linear space of (3.9), and $\hat{z}(s, 1 \mid s-1)$ the projection of $z(s, 1)$ onto the linear space of (3.10). Lemma 3.2 shows that $\varepsilon_{m}(s)(m=2,1)$ is in fact the innovation sequence for the model of (2.1), (3.6).

LEMMA 3.2. The elements of the sequence

$$
\left\{\left.\varepsilon_{2}(s)\right|_{0 \leq s \leq k_{1}} ;\left.\varepsilon_{1}(s)\right|_{k_{1}<s \leq k}\right\}
$$

are mutually uncorrelated, and span the same linear space as (3.2).

PROOF. By virtue of the unbiased property of the projection,

$$
\mathrm{E}\left[\varepsilon_{m}(s)\right]=\mathrm{E}\left[\bar{y}_{m}(s)\right]-\mathrm{E}\left[\hat{\bar{y}}_{m}(s \mid s-1)\right]=0, \quad m=2,1 .
$$

Next, we show that $\varepsilon_{m}(s)$ and $\varepsilon_{l}(\tau)$ are uncorrelated for $s \neq \tau$. In fact, it follows from (3.11) and (3.12) that

$$
\begin{aligned}
& \mathrm{E}\left[\varepsilon_{2}(s) \varepsilon_{l}(\tau)^{\prime}\right]=\bar{H} \mathrm{E}\left[\tilde{z}(s, 2 \mid s-1) \varepsilon_{l}(\tau)^{\prime}\right]+\mathrm{E}\left[\bar{G}_{\theta(s)} \bar{v}(s) \varepsilon_{l}(\tau)^{\prime}\right], \\
& \mathrm{E}\left[\varepsilon_{1}(s) \varepsilon_{l}(\tau)^{\prime}\right]=H \mathrm{E}\left[\tilde{z}(s, 1 \mid s-1) \varepsilon_{l}(\tau)^{\prime}\right]+\mathrm{E}\left[G_{\theta(s)} v(s) \varepsilon_{l}(\tau)^{\prime}\right] .
\end{aligned}
$$

We have $\mathrm{E}\left[\tilde{z}(s, 2 \mid s-1) \varepsilon_{l}(\tau)^{\prime}\right]=0$, since $\tilde{z}(s, 2 \mid s-1)$ is the prediction error of $z(s, 2)$. Meanwhile, $\mathrm{E}\left[\bar{G}_{\theta(s)} \bar{v}(s) \varepsilon_{l}(\tau)^{\prime}\right]=0$, which means that the sequence 
$\left\{\left.\varepsilon_{2}(s)\right|_{0 \leq s \leq k_{1}}\right\}$ is uncorrelated. Similar reasoning shows that $\mathrm{E}\left[\varepsilon_{1}(s) \varepsilon_{l}(\tau)^{\prime}\right]=0$. That is to say, $\left\{\left.\varepsilon_{2}(s)\right|_{0 \leq s \leq k_{1}} ;\left.\varepsilon_{1}(s)\right|_{k_{1}<s \leq k}\right\}$ is an uncorrelated sequence.

Finally, we show that the sequence spans the same linear space as (3.2). It is apparent that $\varepsilon_{2}(s)$ for $s \leq k_{1}$ (or $\varepsilon_{1}(s)$ for $s>k_{1}$ ) is a linear combination of the observations $\bar{y}_{2}(0), \ldots, \bar{y}_{2}(s)$ (or $\bar{y}_{2}(0), \ldots, \bar{y}_{2}\left(k_{1}\right), \bar{y}_{1}\left(k_{1}+1\right), \ldots, \bar{y}_{1}(s)$ ). Conversely, $\bar{y}_{2}(s)$ for $s \leq k_{1}$ (or $\bar{y}_{1}(s), s>k_{1}$ ) can be given in terms of a linear combination of $\varepsilon_{2}(0), \ldots, \varepsilon_{2}(s)$ (or $\left.\varepsilon_{2}(0), \ldots, \varepsilon_{2}\left(k_{1}\right), \varepsilon_{1}\left(k_{1}+1\right), \ldots, \varepsilon_{1}(s)\right)$. This yields the desired results.

Based on Lemma 3.2, the problem of estimating the state $x(s)$ given (3.2) can be converted into the problem of estimating $z(s, 2)$ and $z(s, 1)$ given $(3.13)$, which, due to its orthogonality, can significantly simplify the calculation of the projection.

3.3. Optimal estimator $\hat{\boldsymbol{x}}(\boldsymbol{k} \mid \boldsymbol{k})$ In this subsection we first define matrices associated with the second moment of the above variables. We define

$$
\begin{gathered}
Z(s, \theta)=\mathrm{E}\left[z(s, \theta) z(s, \theta)^{\prime}\right], \quad Z_{i j}(s, \theta)=\mathrm{E}\left[z_{i j}(s, \theta) z_{i j}(s, \theta)^{\prime}\right], \\
\hat{Z}(s, \theta \mid s-1)=\mathrm{E}\left[\hat{z}(s, \theta \mid s-1) \hat{z}(s, \theta \mid s-1)^{\prime}\right],
\end{gathered}
$$

for $\theta=2$ with $0 \leq s \leq k_{1}$ and for $\theta=1$ with $k_{1}<s \leq k$. Then from the definition of $Z_{i j}(s, 2)\left(0 \leq s \leq k_{1}\right)$ and $Z_{i}(s)\left(k_{1}<s \leq k\right)$, the following lemma is obtained.

LEMMA 3.3. For $0 \leq s \leq k_{1}$, the covariance matrix of $z_{i j}(s, 2)$ satisfies the difference equation

$$
\begin{aligned}
Z_{i j}(s+1,2)= & \sum_{l=1}^{N} \sum_{m=1}^{N} \lambda_{l i} \lambda_{m j} A_{l} Z_{l m}(s, 2) A_{l}^{\prime} \\
& +\sum_{l=1}^{N} \sum_{m=1}^{N} \pi_{l}(s) \pi_{m}(s+d) \lambda_{l i} \lambda_{m j} C_{l} C_{l}^{\prime},
\end{aligned}
$$

with the initial value

$$
Z_{i j}(0,2)=\pi_{i}(0) \pi_{j}(d) V, \quad i, j=1, \ldots, N .
$$

For $k_{1}<s \leq k$, the covariance matrix of $z_{i}(s, 1)$ can be calculated by the recursive equation

$$
Z_{i}(s+1,1)=\sum_{l=1}^{N} \lambda_{l i} A_{l} Z_{l}(s, 1) A_{l}^{\prime}+\sum_{l=1}^{N} \pi_{l}(s) \lambda_{l i} C_{l} C_{l}^{\prime},
$$

with initial value

$$
Z_{i}\left(k_{1}+1,1\right)=\sum_{j=1}^{N} Z_{i j}\left(k_{1}+1,2\right), \quad i=1, \ldots, N .
$$

PROOF. For $0 \leq s \leq k_{1}$, in view of

$$
z_{i j}(s+1,2)=x(s+1) \mathbf{1}_{\{\theta(s+1)=i\}} \mathbf{1}_{\{\theta(s+d+1)=j\}}
$$


and (2.1), we obtain

$$
\begin{aligned}
Z_{i j}(s+1,2)= & \mathrm{E}\left[z_{i j}(s+1,2) z_{i j}(s+1,2)^{\prime}\right] \\
= & \mathrm{E}\left[\left(A_{\theta(s)} x(s)+C_{\theta(s)} w(s)\right)\left(A_{\theta(s)} x(s)+C_{\theta(s)} w(s)\right)^{\prime}\right. \\
& \times \mathbf{1}_{\{\theta(s+1)=i\}} \mathbf{1}_{\{\theta(s+d+1)=j\}]} \\
= & \sum_{l=1}^{N} \sum_{m=1}^{N} \lambda_{l i} \lambda_{m j} A_{l} Z_{l m}(s, 2) A_{l}^{\prime} \\
& +\sum_{l=1}^{N} \sum_{m=1}^{N} \pi_{l}(s) \pi_{m}(s+d) \lambda_{l i} \lambda_{m j} C_{l} C_{l}^{\prime} .
\end{aligned}
$$

Recalling that $\mathrm{E}\left[x(0) x(0)^{\prime}\right]=V$, we get the initial covariance matrix $Z_{i j}(0,2)=$ $\pi_{i}(0) \pi_{j}(d) V$.

Similarly, from the definition of $z_{i}(s, 1)$ and System (2.1), we get the recursive difference equation (3.15) with initial value given by (3.16). The proof is complete.

In what follows, we present the Riccati difference equations $P(s, 2 \mid s-1)$ and $P(s, 1 \mid s-1)$.

THEOREM 3.4. For a given time instant $k$, the covariance matrices $P(s, i \mid s-1)(i=$ $2,1)$ can be calculated as follows:

- With the initial value $P(0,2 \mid-1)=\operatorname{diag}\left\{\pi_{i}(0) \pi_{j}(d) V\right\}$, the matrix $P(s, 2 \mid s-1)$, for $0 \leq s \leq k_{1}$, can be obtained by solving the Riccati equation

$$
\begin{aligned}
P(s+ & 1,2 \mid s) \\
= & \overline{\mathcal{A}} P(s, 2 \mid s-1) \overline{\mathcal{A}}^{\prime}+\operatorname{diag}\left\{\sum_{l=1}^{N} \sum_{m=1}^{N} \pi_{l}(s) \pi_{m}(s+d) \lambda_{l i} \lambda_{m j} C_{l} C_{l}^{\prime}\right\} \\
& -\overline{\mathcal{A}} K_{2}(s) \bar{H} P(s, 2 \mid s-1) \overline{\mathcal{A}}^{\prime}+\mathcal{B}(Z(s, 2)),
\end{aligned}
$$

where

$$
\begin{gathered}
\overline{\mathcal{A}}=\left(\Lambda^{\prime} \otimes I_{n} \cdot \operatorname{diag}\left\{A_{1}, \ldots, A_{N}\right\}\right) \otimes \Lambda^{\prime}, \\
K_{2}(s)=P(s, 2 \mid s-1) \bar{H}^{\prime}\left(\bar{H} P(s, 2 \mid s-1) \bar{H}^{\prime}+\bar{G}(s) \bar{G}(s)^{\prime}\right)^{-1}, \\
\mathcal{B}(Z(s, 2))=\operatorname{diag}\left\{\sum_{l=1}^{N} \sum_{m=1}^{N} \lambda_{l i} \lambda_{m j} A_{l} Z_{l m}(s, 2) A_{l}^{\prime}\right\}-\overline{\mathcal{A}} Z(s, 2) \overline{\mathcal{A}}^{\prime} .
\end{gathered}
$$

- For $k_{1}<s \leq k$, the matrix $P(s, 1 \mid s-1)$ can be calculated recursively by

$$
\begin{aligned}
P(s+1,1 \mid s)= & \mathcal{A} P(s, 1 \mid s-1) \mathcal{A}^{\prime}+\operatorname{diag}\left\{\sum_{l=1}^{N} \pi_{l}(s) \lambda_{l i} C_{l} C_{l}^{\prime}\right\} \\
& -\mathcal{A} K_{1}(s) H P(s, 1 \mid s-1) \mathcal{A}^{\prime}+\mathcal{B}(Z(s, 1)), \\
P\left(k_{1}+1,1 \mid k_{1}\right)= & \operatorname{diag}\left\{\sum_{j=1}^{N} P_{i j}\left(k_{1}+1,2 \mid k_{1}\right)\right\},
\end{aligned}
$$


where

$$
\begin{gathered}
\mathcal{A}=\Lambda^{\prime} \otimes I_{n} \cdot \operatorname{diag}\left\{A_{1}, \ldots, A_{N}\right\}, \\
K_{1}(s)=P(s, 1 \mid s-1) H^{\prime}\left(H P(s, 1 \mid s-1) H^{\prime}+G(s) G(s)^{\prime}\right)^{-1}, \\
\mathcal{B}(Z(s, 1))=\operatorname{diag}\left\{\sum_{l=1}^{N} \lambda_{l i} A_{l} Z_{l}(s, 1) A_{l}^{\prime}\right\}-\mathcal{A} Z(s, 1) \mathcal{A}^{\prime} .
\end{gathered}
$$

Proof. The proof is divided into two stages. Assume initially that $\mathrm{E}[z(0,2)]=0$; then all random variables have zero expectation.

Firstly, for $0 \leq s \leq k_{1}$, based on (2.4) and (2.5) the LMMSE estimator $\hat{z}(s, 2 \mid s-1)$ satisfies

$$
\begin{gathered}
\hat{z}(s, 2 \mid s-1)=\mathrm{E}\left[z(s, 2)\left(\left(\bar{y}_{2}\right)^{s-1}\right)^{\prime}\right] \operatorname{cov}\left(\left(\bar{y}_{2}\right)^{s-1}\right)^{-1}\left(\bar{y}_{2}\right)^{s-1}, \\
\hat{z}(s, 2 \mid s)=\hat{z}(s, 2 \mid s-1)+\mathrm{E}\left[z(s, 2) \varepsilon_{2}(s)^{\prime}\right] \mathrm{E}\left[\varepsilon_{2}(s) \varepsilon_{2}(s)^{\prime}\right]^{-1}\left(\bar{y}_{2}(s)-\hat{\bar{y}}_{2}(s \mid s-1)\right),
\end{gathered}
$$

where $\left(\bar{y}_{2}\right)^{s-1}=\operatorname{col}\left\{\bar{y}_{2}(0), \ldots, \bar{y}_{2}(s-1)\right\}$.

It has been shown that

$$
\bar{y}_{2}(s)=\bar{H} z(s, 2)+\bar{G}_{\theta(s)} \bar{v}(s),
$$

and the corresponding innovation sequence can be given as

$$
\varepsilon_{2}(s)=\bar{H} \tilde{z}(s, 2 \mid s-1)+\bar{G}_{\theta(s)} \bar{v}(s) .
$$

From the independence of $v(s), v_{1}(s)$ and $\theta(s),\left(\bar{y}_{2}\right)^{s-1}$, we obtain that

$$
\begin{aligned}
& \left\langle\beta^{\prime} \bar{G}_{\theta(s)} \bar{v}(s) ; \alpha^{\prime}\left(\bar{y}_{2}\right)^{s-1}\right\rangle \\
& =\mathrm{E}\left\{\left[v(s)^{\prime} G_{\theta(s)}^{\prime} v_{1}(s+d)^{\prime} \tilde{G}_{\theta(s+d)}^{\prime}\right]^{\prime} \beta \alpha^{\prime}\left(\bar{y}_{2}\right)^{s-1}\right\} \\
& =\beta \alpha^{\prime}\left[\mathrm{E}\left(v(s)^{\prime} G_{\theta(s)}^{\prime}\left(\bar{y}_{2}\right)^{s-1}\right) \mathrm{E}\left(v_{1}(s+d)^{\prime} \tilde{G}_{\theta(s+d)}^{\prime}\left(\bar{y}_{2}\right)^{s-1}\right)\right]=0,
\end{aligned}
$$

which shows that $\bar{G}_{\theta(s)} \bar{v}(s)$ is orthogonal to $\mathcal{L}\left\{\left.\bar{y}_{2}(\tau)\right|_{0 \leq \tau \leq s-1}\right\}$. Similar reasoning indicates the orthogonality between $\tilde{z}(s, 2 \mid s-1)$ and $\bar{G}_{\theta(s)} \overline{\bar{v}}(s)$. Recalling that

$$
\hat{z}(s, 2 \mid s-1) \in \mathcal{L}\left\{\left.\bar{y}_{2}(\tau)\right|_{0 \leq \tau \leq s-1}\right\}
$$

and $\tilde{z}(s, 2 \mid s-1)$ is orthogonal to $\mathcal{L}\left\{\left.\bar{y}_{2}(\tau)\right|_{0 \leq \tau \leq s-1}\right\}$, we obtain that $\hat{z}(s, 2 \mid s-1)$ is orthogonal to $\tilde{z}(s, 2 \mid s-1)$. Then from (3.21) and (3.22), we get that

$$
\begin{gathered}
\mathrm{E}\left[\varepsilon_{2}(s) \varepsilon_{2}(s)^{\prime}\right]=\bar{H} P(s, 2 \mid s-1) \bar{H}^{\prime}+\bar{G}(s) \bar{G}(s)^{\prime}, \\
\mathrm{E}\left[z(s, 2) \varepsilon_{2}(s)^{\prime}\right]=\mathrm{E}\left[(\tilde{z}(s, 2 \mid s-1)+\hat{z}(s, 2 \mid s-1)) \varepsilon_{2}(s)^{\prime}\right]=P(s, 2 \mid s-1) \bar{H}^{\prime} .
\end{gathered}
$$


Recalling that $\mathrm{E}\left[\bar{y}_{2}(s)\right]=0$ and defining $\mathcal{Y}_{2}^{s-1}=\operatorname{cov}\left(\left(\bar{y}_{2}\right)^{s-1}\right)^{-1}\left(\bar{y}_{2}\right)^{s-1}$, we obtain from (3.19) that

$$
\begin{aligned}
\hat{z}_{i j}(s, 2 \mid s-1) \\
\quad=\mathrm{E}\left[z_{i j}(s, 2)\left(\left(\bar{y}_{2}\right)^{s-1}\right)^{\prime}\right] \mathcal{Y}_{2}^{s-1}, \\
=\mathrm{E}\left[x(s) \mathbf{1}_{\{\theta(s)=i\}} \mathbf{1}_{\{\theta(s+d)=j\}}\left(\left(\bar{y}_{2}\right)^{s-1}\right)^{\prime}\right] \mathcal{Y}_{2}^{s-1}, \\
=\mathrm{E}\left\{\mathrm{E}\left[A_{\theta(s-1)} x(s-1) \mathbf{1}_{\{\theta(s)=i\}} \mathbf{1}_{\{\theta(s+d)=j\}}\left(\left(\bar{y}_{2}\right)^{s-1}\right)^{\prime} \mid \mathcal{F}_{s+d-1}\right]\right\} \mathcal{Y}_{2}^{s-1}, \\
=\mathrm{E}\left\{\sum_{m=1}^{N} \lambda_{m j} \mathrm{E}\left[A_{\theta(s-1)} x(s-1) \mathbf{1}_{\{\theta(s)=i\}} \mathbf{1}_{\{\theta(s+d-1)=m\}}\left(\left(\bar{y}_{2}\right)^{s-1}\right)^{\prime} \mid \mathcal{F}_{s-1}\right]\right\} \mathcal{Y}_{2}^{s-1}, \\
=\sum_{l=1}^{N} \sum_{m=1}^{N} \lambda_{l i} \lambda_{m j} A_{l} \hat{z}_{l m}(s-1,2 \mid s-1),
\end{aligned}
$$

where we have used the fact that $\operatorname{cov}\left(\left(\bar{y}_{2}\right)^{s-1}\right)^{-1}>0\left(0 \leq s \leq k_{1}\right)$ (see [9]), and in the third equality above we have used the fact that $C_{\theta(s)} w(s-1)$ and $\left(\left(\bar{y}_{2}\right)^{s-1}\right)$ are orthogonal (by the same reasoning as in (3.23) above).

In view of (3.20), (3.24) and (3.25), we obtain the recursive equation of the linear mean-square filter

$$
\begin{aligned}
\hat{z}(s, 2 \mid s) & =\hat{z}(s, 2 \mid s-1)+\mathrm{E}\left[z(s, 2) \varepsilon_{2}(s)^{\prime}\right] \mathrm{E}\left[\varepsilon_{2}(s) \varepsilon_{2}(s)^{\prime}\right]^{-1}\left(\bar{y}_{2}(s)-\hat{\bar{y}}_{2}(s \mid s-1)\right) \\
& =\hat{z}(s, 2 \mid s-1)+K_{2}(s)\left(\bar{y}_{2}(s)-\bar{H} \hat{z}(s, 2 \mid s-1)\right),
\end{aligned}
$$

where

$$
K_{2}(s)=P(s, 2 \mid s-1) \bar{H}^{\prime}\left(\bar{H} P(s, 2 \mid s-1) \bar{H}^{\prime}+\bar{G}(s) \bar{G}(s)^{\prime}\right)^{-1} .
$$

In light of (3.26), we can obtain

$$
\hat{z}(s, 2 \mid s-1)=\overline{\mathcal{A}} \hat{z}(s-1,2 \mid s-1) .
$$

Noting that

$$
\bar{y}_{2}(s)-\bar{H} \hat{z}(s, 2 \mid s-1)=\bar{H} \tilde{z}(s, 2 \mid s-1)+\bar{G}_{\theta(s)} \bar{v}(s),
$$

and $\hat{z}(s, 2 \mid s-1), \tilde{z}(s, 2 \mid s-1)$ and $\bar{G}_{\theta(s)} \bar{v}(s)$ are mutually orthogonal, then we obtain

$$
\begin{aligned}
& \mathrm{E}\left[\hat{z}(s, 2 \mid s) \hat{z}(s, 2 \mid s)^{\prime}\right] \\
&=\mathrm{E}\left\{\left[\hat{z}(s, 2 \mid s-1)+K_{2}(s)\left(\bar{H} \tilde{z}(s, 2 \mid s-1)+\bar{G}_{\theta(s)} \bar{v}(s)\right)\right]\right. \\
&\left.\times\left[\hat{z}(s, 2 \mid s-1)+K_{2}(s)\left(\bar{H} \tilde{z}(s, 2 \mid s-1)+\bar{G}_{\theta(s)} \bar{v}(s)\right)\right]^{\prime}\right\} \\
&= \hat{Z}(s, 2 \mid s-1)+K_{2}(s)\left[\bar{H} P_{2}(s \mid s-1) \bar{H}^{\prime}+\bar{G}(s) \bar{G}(s)^{\prime}\right] K_{2}(s)^{\prime} \\
&= \hat{Z}(s, 2 \mid s-1)+K_{2}(s) \bar{H} P(s, 2 \mid s-1) .
\end{aligned}
$$


In view of (3.27),

$$
\begin{aligned}
& \hat{Z}(s, 2 \mid s-1)=\overline{\mathcal{A}} \hat{Z}(s-1,2 \mid s-1) \overline{\mathcal{A}}^{\prime} \\
& \hat{Z}(0,2 \mid s-1)=\mathrm{E}(\hat{z}(0,2 \mid s-1)) \mathrm{E}\left(\hat{z}(0,2 \mid s-1)^{\prime}\right)=0 .
\end{aligned}
$$

Meanwhile, in view of Lemma 3.3, (3.14) can be reformulated as

$$
\begin{aligned}
Z(s+1,2)= & \operatorname{diag}\left\{\sum_{l=1}^{N} \sum_{m=1}^{N} \pi_{l}(s) \pi_{m}(s+d) \lambda_{l i} \lambda_{m j} C_{l} C_{l}^{\prime}\right\}+\overline{\mathcal{A}} Z(s, 2) \overline{\mathcal{A}}^{\prime} \\
& +\mathcal{B}(Z(s, 2))
\end{aligned}
$$

where

$$
\mathcal{B}(Z(s, 2))=\operatorname{diag}\left\{\sum_{l=1}^{N} \sum_{m=1}^{N} \lambda_{l i} \lambda_{m j} A_{l} Z_{l m}(s, 2) A_{l}^{\prime}\right\}-\overline{\mathcal{A}} Z(s, 2) \overline{\mathcal{A}}^{\prime},
$$

and the initial value $Z(0,2)=\operatorname{diag}\left\{\pi_{i}(0) \pi_{j}(d) V\right\}$.

Considering (3.28)-(3.30), and noticing that

$$
P(s, 2 \mid s-1)=Z(s, 2)-\hat{Z}(s, 2 \mid s-1),
$$

we obtain that

$$
\begin{aligned}
P(s+ & 1,2 \mid s) \\
= & \overline{\mathcal{A}} P(s, 2 \mid s-1) \overline{\mathcal{A}}^{\prime}+\operatorname{diag}\left\{\sum_{l=1}^{N} \sum_{m=1}^{N} \pi_{l}(s) \pi_{m}(s+d) \lambda_{l i} \lambda_{m j} C_{l} C_{l}^{\prime}\right\} \\
& -\overline{\mathcal{A}} K_{2}(s) \bar{H} P(s, 2 \mid s-1) \overline{\mathcal{A}}^{\prime}+\mathcal{B}(Z(s, 2)),
\end{aligned}
$$

with $P(0,2 \mid-1)=\operatorname{diag}\left\{\pi_{i}(0) \pi_{j}(d) V\right\}$.

In the second part of the proof, for $k_{1}<s \leq k$ we derive the Riccati equation for $P(s, 1 \mid s-1)$. Following the same steps as above, we obtain that

$$
\begin{gathered}
\hat{z}(s, 1 \mid s)=\hat{z}(s, 1 \mid s-1)+K_{1}(s)\left(\bar{y}_{1}(s)-H \hat{z}(s, 1 \mid s-1)\right), \\
\hat{z}(s, 1 \mid s-1)=\mathcal{A} \hat{z}(s-1,1 \mid s-1),
\end{gathered}
$$

where

$$
\begin{gathered}
\mathcal{A}=\Lambda^{\prime} \otimes I_{n} \cdot \operatorname{diag}\left\{A_{1}, \ldots, A_{N}\right\} \\
K_{1}(s)=P(s, 1 \mid s-1) H^{\prime}\left(H P(s, 1 \mid s-1) H^{\prime}+G(s) G(s)^{\prime}\right)^{-1} .
\end{gathered}
$$

According to (3.31) and (3.32), the corresponding covariance matrices are

$$
\begin{gathered}
\hat{Z}(s, 1 \mid s)=\hat{Z}(s, 1 \mid s-1)+K_{1}(s) H P(s, 1 \mid s-1), \\
\hat{Z}(s, 1 \mid s-1)=\mathcal{A} \hat{Z}(s-1,2 \mid s-1) \mathcal{A}^{\prime} .
\end{gathered}
$$


Recalling the definition of $Z(s, 1),(3.15)$ can be written as

$$
Z(s+1,1)=\mathcal{A} Z(s, 1) \mathcal{A}^{\prime}+\mathcal{B}(Z(s, 1))+\operatorname{diag}\left\{\sum_{l=1}^{N} \pi_{l}(s) \lambda_{l i} C_{l} C_{l}^{\prime}\right\},
$$

where

$$
\begin{gathered}
\mathcal{B}(Z(s, 1))=\operatorname{diag}\left\{\sum_{l=1}^{N} \lambda_{l i} A_{l} Z_{l}(s, 1) A_{l}^{\prime}\right\}-\mathcal{A} Z(s, 1) \mathcal{A}^{\prime}, \\
Z\left(k_{1}+1,1\right)=\operatorname{diag}\left\{\sum_{j=1}^{N} Z_{i j}\left(k_{1}+1,2\right)\right\} .
\end{gathered}
$$

In light of (3.33)-(3.35), we get that

$$
\begin{aligned}
P(s+1,1 \mid s)= & \mathcal{A} P(s, 1 \mid s-1) \mathcal{A}^{\prime}+\operatorname{diag}\left\{\sum_{l=1}^{N} \pi_{l}(s) \lambda_{l i} C_{l} C_{l}^{\prime}\right\} \\
& -\mathcal{A} K_{1}(s) H P(s, 1 \mid s-1) \mathcal{A}^{\prime}+\mathcal{B}(Z(s, 1)),
\end{aligned}
$$

with the initial value

$$
P\left(k_{1}+1,1 \mid k_{1}\right)=\operatorname{diag}\left\{\sum_{j=1}^{N} P_{i j}\left(k_{1}+1,2 \mid k_{1}\right)\right\} .
$$

The desired result is obtained.

In Theorem 3.4 we write $P(s, 2 \mid s-1)$ and $P(s, 1 \mid s-1)$ directly as two Riccati equations with additional terms that depend on the second moment matrix of the state variable. In the following, we shall give the solution to the optimal filtering problem by applying the geometric argument analysis and the Riccati equations obtained above.

THEOREM 3.5. Consider (2.1)-(2.3); the optimal linear mean-square error estimator $\hat{x}(k \mid k)$ is given by

$$
\hat{x}(k \mid k)=\sum_{i=1}^{N} \hat{z}_{i}(k, 1 \mid k),
$$

where the estimator $\hat{z}_{i}(k, 1 \mid k)$ (the ith element of the vector $\left.\hat{z}(k, 1 \mid k)\right)$ is computed following the steps below.

- For $0 \leq s \leq k_{1}, \hat{z}(s, 2 \mid s)$ can be calculated by the following difference equation with the initial value $\hat{z}(0,2 \mid-1)=\mathrm{E}(z(0))=0$ :

$$
\begin{aligned}
\hat{z}(s, 2 \mid s) & =\hat{z}(s, 2 \mid s-1)+K_{2}(s)\left(\bar{y}_{2}(s)-\bar{H} \hat{z}(s, 2 \mid s-1)\right), \\
\hat{z}(s, 2 \mid s-1) & =\overline{\mathcal{A}} \hat{z}(s-1,2 \mid s-1),
\end{aligned}
$$

where $K_{2}(s)$ can be obtained by (3.17). 
- For $k_{1}<s \leq k, \hat{z}(s, 1 \mid s)$ can be computed by the recursive equation

$$
\begin{aligned}
\hat{z}(s, 1 \mid s) & =\hat{z}(s, 1 \mid s-1)+K_{1}(s)\left(\bar{y}_{1}(s)-H \hat{z}(s, 1 \mid s-1)\right), \\
\hat{z}(s, 1 \mid s-1) & =\mathcal{A} \hat{z}(s-1,1 \mid s-1),
\end{aligned}
$$

where $K_{1}(s)$ can be calculated by (3.18), and the initial state is

$$
\hat{z}\left(k_{1}+1 \mid k_{1}\right)=\operatorname{col}\left\{\sum_{j=1}^{N} z_{1 j}\left(k_{1}+1,2 \mid k_{1}\right), \ldots, \sum_{j=1}^{N} \hat{z}_{N j}\left(k_{1}+1,2 \mid k_{1}\right)\right\} .
$$

PROOF. The proof of this theorem is obvious, and is omitted here.

In Theorem 3.5 we first give the solution to the optimal filter $\hat{z}(s, 2 \mid s)\left(0 \leq s \leq k_{1}\right)$ and $\hat{z}(s, 1 \mid s)\left(k_{1}<s \leq k\right)$, then on the basis of the Markovian jump property, the state estimator $\hat{x}(k \mid k)$ is obtained.

REMARK 1. In the above discussion, we assume that $E(x(0))=0$. It can be shown that (3.36)-(3.41) are also satisfied if $E(x(0))=\mu \neq 0$.

REMARK 2. When $0 \leq k \leq d$, the optimal estimator $\hat{x}(k \mid k)$ is indeed the projection of $x(k)$ onto the linear space of $\left\{\left.y(s)\right|_{0 \leq s \leq k}\right\}$. Note that the observation sequence $y(s)$ is delay-free, and thus the estimation problem is reduced to a linear mean-square estimator for Systems (2.1) and (2.2), which has been considered in [18].

\section{Numerical simulations}

In this section we apply our algorithm to a very simple illustrative example and show that the algorithm offers a powerful tool in several problems, such as maneuvering target tracking using a wireless sensor network where communication and link delays are often encountered. Assume a target moving in two-dimensional space according to the standard constant acceleration model [2, 13]. This model assumes that the vehicle has constant acceleration equal to zero except for a small perturbation. Denote the position of the vehicle in the two dimensions by $p_{x}$ and $p_{y}$, the velocities by $v_{x}$ and $v_{y}$. Here we use two groups of sensors, labelled 1 and 2, for the target tracking and each of them has two sensors that can collect the information of the maneuvering target correctly. At each time $k$, only one group is used to collect the target information, and the transition between the two groups is determined by a twostate Markov chain $\theta(k)$. It is assumed that $\theta(k) \in\{1,2\}$ is a first-order, homogeneous Markov process with the probability transition matrix $\Lambda=\left[\left(\lambda_{i j}\right)\right]$ given by

$$
\Lambda=\left[\begin{array}{ll}
0.85 & 0.15 \\
0.70 & 0.30
\end{array}\right]
$$

The initial mode probabilities of $\theta(k)$ are

$$
\pi_{1}(0)=\mathcal{P}(\theta(0)=1)=0.5 \quad \text { and } \quad \pi_{2}(0)=\mathcal{P}(\theta(0)=2)=0.5 .
$$


The dynamic model of the target is as shown in [2] with parameters

$$
A_{1}=A_{2}=\left[\begin{array}{llll}
1 & 0 & h & 0 \\
0 & 1 & 0 & h \\
0 & 0 & 1 & 0 \\
0 & 0 & 0 & 1
\end{array}\right], \quad C_{1}=C_{2}=\left[\begin{array}{cc}
h^{2} / 2 & 0 \\
0 & h^{2} / 2 \\
h & 0 \\
0 & h
\end{array}\right], \quad x=\left[\begin{array}{c}
p_{x} \\
p_{y} \\
v_{x} \\
v_{y}
\end{array}\right]
$$

where $h$ is the discretization step size in this simulation and set to $0.1 \mathrm{~s}$. The term $w(k)$ is considered as the perturbation term in acceleration. In the numerical example these sensors are only used to gather the location information of the maneuvering target. Moreover, in each group one sensor can gather the target information instantaneously, while the other is delayed with lag $d$. For this model, the measurements taken by the two sensors in each group are given respectively as

$$
\begin{aligned}
y(k) & =\left[\begin{array}{llll}
1 & 0 & 0 & 0 \\
0 & 1 & 0 & 0
\end{array}\right] x(k)+G_{\theta(k)} v(k), \\
y_{1}(k) & =\left[\begin{array}{llll}
1 & 0 & 0 & 0 \\
0 & 1 & 0 & 0
\end{array}\right] x(k-d)+\tilde{G}_{\theta(k)} v_{1}(k),
\end{aligned}
$$

where

$$
G_{1}=\left[\begin{array}{cc}
2.4 & 0 \\
0 & 2.4
\end{array}\right], \quad G_{2}=\left[\begin{array}{cc}
0.4 & 0 \\
0 & 0.4
\end{array}\right], \quad \tilde{G}_{1}=\left[\begin{array}{cc}
0.7 & 0 \\
0 & 0.7
\end{array}\right], \quad \tilde{G}_{2}=\left[\begin{array}{cc}
1.4 & 0 \\
0 & 1.4
\end{array}\right] .
$$

The time delay $d$ is set to 10 and the time horizon $N$ is set to 200. The initial state $x(0)$ is a random variable with $\mathrm{E}(x(0))=0$ and

$$
\mathrm{E}\left\{x(0) x(0)^{\prime}\right\}=\left[\begin{array}{llll}
1 & 0 & 0 & 0 \\
0 & 1 & 0 & 0 \\
0 & 0 & 1 & 0 \\
0 & 0 & 0 & 1
\end{array}\right]
$$

In the actual system we use $p_{x}(0)=3, p_{y}(0)=3, v_{x}(0)=0.8$ and $v_{y}(0)=0.4$ for all simulation runs to generate $x(k)$. In the simulation we run 50 Monte Carlo simulations from $k=0$ to $N$. To make the effect of mode changes evident, all simulations were based on the same Markov chain sample path.

Following the design procedures as in Section 3, we obtain the simulation results as follows. In Figure 1 we show the true and estimated position values of $p_{x}$ and $p_{y}$. The solid line represents the true position and the dashed line shows the estimate of the target trajectory. Figure 1 shows that the estimated values are very close to the true positions throughout the entire period of tracking. Figure 2 shows root mean-square (RMS) errors of the positions. We note that the RMS position errors of the filters are within 0.5 . Therefore, the response of the proposed filter to the maneuvering target is fairly good. 

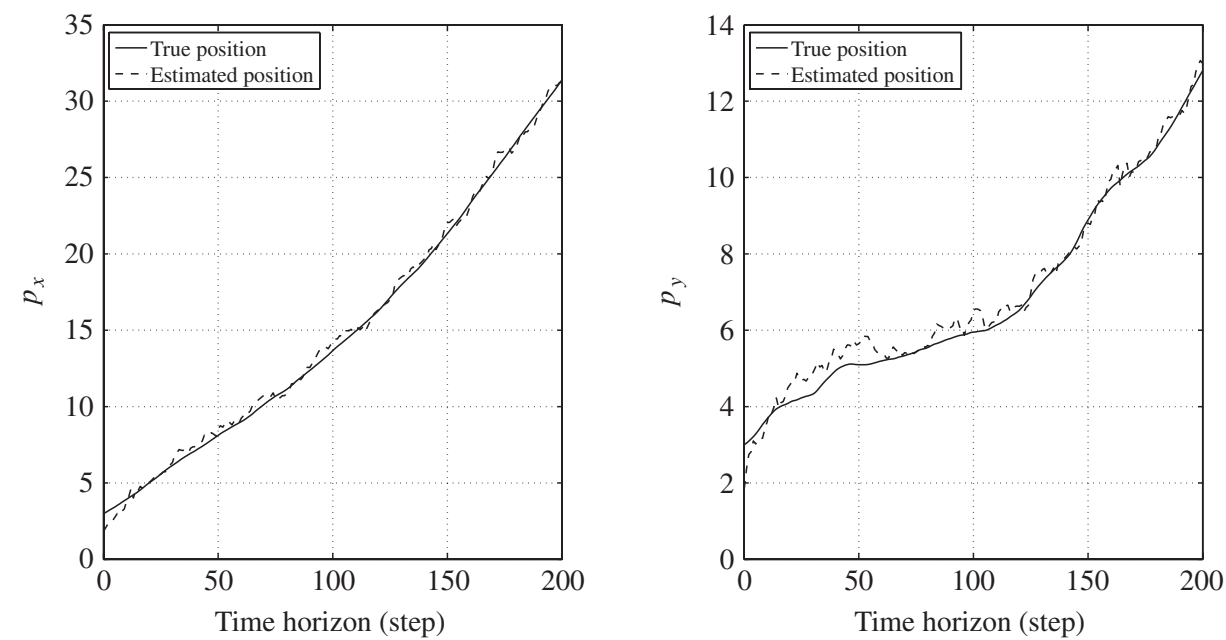

FIGURE 1. The position of the target in the $x$ direction (left) and the $y$ direction (right). The true position is shown by a solid line and estimated position by a dashed line.
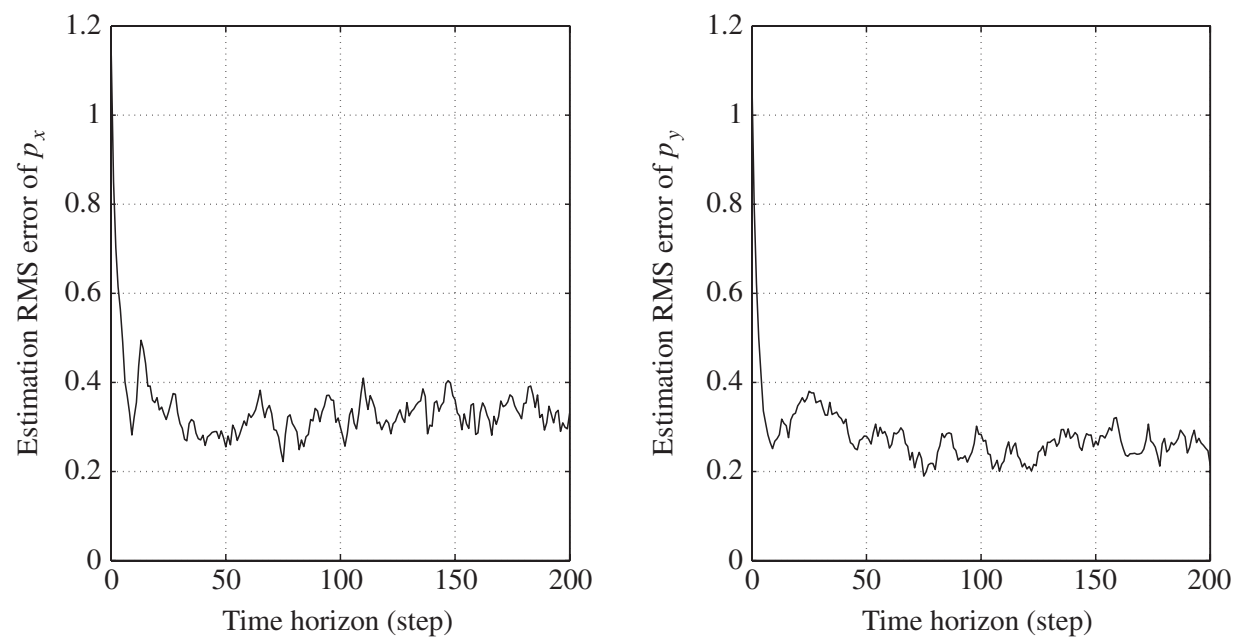

FIGURE 2. Estimation RMS error of the target in the $x$ direction (left) and the $y$ direction (right).

\section{Conclusions}

This paper has addressed the LMMSE estimation for discrete-time MJLSs with delayed measurements. The solution was given in terms of Riccati difference equations. The key to our development in the estimation is to apply the reorganized innovation analysis method to treat the measurement delay. It is worth highlighting that the results of this paper are applicable to the estimation problem of systems with multiple observation delays. 


\section{References}

[1] G. A. Ackerson and K. S. Fu, "On state estimation in switching environments", IEEE Trans. Automat. Control 15 (1970) 10-17.

[2] Y. Bar-Shalom, X. R. Li and T. Kirubarajan, Estimation with applications to tracking and navigation: theory, algorithms and software (Wiley, New York, 2001).

[3] M. V. Basin, M. A. Alcorta García and J. G. Rodríguez González, "Optimal filtering for linear systems with state and observation delays", J. Robust Nonlinear Control 15 (2004) 859-871.

[4] M. V. Basin and R. Martínez Zúniga, "Optimal linear filtering over observations with multiple delays", J. Robust Nonlinear Control 14 (2004) 685-696.

[5] K. Benjelloun and E. K. Boukas, "Mean square stochastic stability of linear time-delay system with Markovian jumping parameters", IEEE Trans. Automat. Control 43 (1998) 1456-1460.

[6] H. A. P. Blom and Y. Bar-Shalom, "The interacting multiple model algorithm for systems with Markov switching coefficients", IEEE Trans. Automat. Control 33 (1988) 780-783.

[7] Y. Boers and J. N. Driessen, "Interacting multiple model particle filter", IEE Proc. Radar Sonar Navigation 150 (2003) 344-349.

[8] E. K. Boukas and Z. K. Liu, "Robust $H_{\infty}$ filtering for polytopic uncertain time-delay systems with Markov jumps", Comput. Electr. Eng. 28 (2002) 171-193.

[9] O. L. V. Costa, "Linear minimum mean square error estimation for discrete-time Markovian jump linear systems", IEEE Trans. Automat. Control 39 (1994) 1685-1689.

[10] O. L. V. Costa and W. L. de Paulo, "Indefinite quadratic with linear costs optimal control of Markov jump with multiplicative noise systems", Automatica 43 (2007) 587-597.

[11] O. L. V. Costa and S. Guerra, "Stationary filter for linear minimum mean square error estimator of discrete-time Markovian jump systems", IEEE Trans. Automat. Control 47 (2002) 1351-1356.

[12] M. H. A. Davis and R. B. Vinter, Stochastic modelling and control (Chapman \& Hall, London, 1985).

[13] V. Gupta, T. H. Chung, B. Hassibi and R. M. Murray, "On a stochastic sensor selection algorithm with applications in sensor scheduling and sensor coverage", Automatica 42 (2006) 251-260.

[14] A. Logothetis and V. Krishnamurthy, "Expectation maximization algorithms for map estimation of jump Markov linear systems", IEEE Trans. Signal Process. 47 (1999) 2139-2156.

[15] M. S. Mahmoud and P. Shi, "Robust Kalman filtering for continuous time-lag systems with Markovian jump parameters", IEEE Trans. Circuits Syst. I 50 (2003) 98-105.

[16] Z. Wang, J. Lam and X. Liu, "Robust filtering for discrete-time Markovian jump delay systems", IEEE Signal Process. Lett. 11 (2004) 659-662.

[17] H. Zhang, X. Lu and D. Cheng, "Optimal estimation for continuous-time systems with delayed measurements", IEEE Trans. Automat. Control 51 (2006) 823-827.

[18] H. Zhang, L. Xie and D. Zhang, "A reorganized innovation approach to linear estimation", IEEE Trans. Automat. Control 49 (2004) 1810-1814. 